AYOS, Emilio; DALLORSO, Nicolás. "(In)seguridad y condiciones de vida en la problematización de la cuestión social: Políticas sociales y políticas de prevención social del delito".

Polit. crim. Vol. 6, № 11 (Junio 2011), Art. 1, pp. 1 - 18.

[http://www.politicacriminal.cl/Vol_06/n_11/Vol6N11A1.pdf]

\title{
(In)seguridad y condiciones de vida en la problematización de la cuestión social: Políticas sociales y políticas de prevención social del delito.
}

\author{
Emilio J. Ayos * \\ Nicolás Dallorso **
}

\section{Resumen}

En el presente artículo buscamos indagar en las articulaciones que se presentan en la coyuntura sociopolítica actual de la Argentina entre las formas de problematización de la cuestión social, las políticas preventivas de control del delito y las reorientaciones en materia de políticas sociales. Entendemos que en la última década se ha consolidado en la Argentina una tendencia que construye progresivamente a la problemática de la (in)seguridad como núcleo de esa tensión constante propia de las sociedades capitalistas, la cuestión social. Esta particular problematización de la cuestión social, que presenta al problema de la (in)seguridad como expresión hegemónica del modo en que es interpretada, diagnosticada y encauzada la potencial fractura social, brinda las condiciones de emergencia de nuevas intervenciones que construyen específicas relaciones entre las nociones de "delito" y "condiciones de vida". En el campo de las políticas sociales hemos observado una mutación relevante en la fundamentación de las intervenciones sociales que han abandonado un paradigma vinculado a la protección integral de las condiciones de vida de la masa de trabajadores y han adoptado una disposición tendencial a privilegiar la fundamentación de intervenciones de carácter puntual y coyuntural sobre grupos clasificados de acuerdo a sus niveles de riesgo y peligrosidad. Asimismo, la reorientación de la política criminal hacia lo social aparece como uno de los lugares fundamentales para observar estos procesos de redefinición de la cuestión social, haciendo hincapié en el carácter fundamentalmente problemático de la relación entre estrategias preventivas y políticas sociales.

\section{Palabras clave}

Cuestión social, inseguridad, política social, prevención social del delito

\footnotetext{
* Licenciado en Sociología, Especialista en Planificación y Gestión de Políticas Sociales (FSOC-UBA) y Magíster en Políticas Sociales (FSOC-UBA). Docente en la Facultad de Ciencias Sociales de la Universidad de Buenos Aires. Becario doctoral del Consejo de Investigaciones Científicas y Técnicas (CONICET). Integrante del Programa de Estudios del Control Social y del Grupo de Estudio sobre Políticas Sociales y Condiciones de Trabajo, ambos en el Instituto de Investigaciones Gino Germani (UBA). Dirección: calle Brasil No 410 3ro. "C", Ciudad Autónoma de Buenos Aires. Teléfono: 32212714. Correo electrónico: emilio.ayos@gmail.com

** Licenciado en Ciencia Política, Especialista en Planificación y Gestión de Políticas Sociales, Magíster en Políticas Sociales y Doctor en Ciencias Sociales (FSOC-UBA). Docente en la Facultad de Ciencias Sociales de la Universidad de Buenos Aires. Becario posdoctoral del Consejo de Investigaciones Científicas y Técnicas (CONICET). Integrante del Programa de Estudios del Control Social en el Instituto de Investigaciones Gino Germani (UBA). Dirección: Tte. Gral. Juan D. Perón No 2581 4to. "H", Ciudad Autónoma de Buenos Aires. Teléfono: 3968-8660. Correo electrónico: nicolas_dallorso@yahoo.com.ar
} 
AYOS, Emilio; DALLORSO, Nicolás. "(In)seguridad y condiciones de vida en la problematización de la cuestión social: Políticas sociales y políticas de prevención social del delito".

\begin{abstract}
In this article we seek to investigate the articulations that take place in Argentina's current socio-political situation between the different forms in which the social question is problematized, the preventive politics of crime control and the reorientations in social policy field. We assume that during the last decade, in Argentina, a tendency to construct the insecurity problem as the core of the constant tension of capitalistic societies has been progressively consolidated. This particular problematization of the social question, which shows the insecurity problem as the hegemonic expression of the way in which potential social fractures are interpreted, diagnosed, and conducted offers the conditions of emergence of new interventions that construct specific relations between the notions of "crime" and "living conditions". In social policy field, we have recognized an important mutation in the foundation of social interventions that have abandoned a paradigm that hold the integral protection of the living conditions of a working mass and have adopted an orientation that privileges the foundation of specific and temporary interventions on groups classified by risk and danger levels. In addition, the reorientation of criminal policy to the social issue appears as one of the privileged places to observe these processes of redefinition of the social question. We emphasize the problematic relation between preventive strategies of criminal control and social policies.
\end{abstract}

\title{
Key words
}

Social question, insecurity, social policy, social prevention of crimes

\section{Introducción.}

En los últimos veinte años, en la Argentina se hicieron visibles en diversos ámbitos de la vida social claros efectos de la transformación que implicó la desestructuración de los arreglos institucionales welfaristas y la erosión paulatina de la sociedad salarial, tal como sucedió en la mayoría de las sociedades capitalistas occidentales. Si bien, dada la magnitud de esta transformación, es imposible abordar, en este artículo, con profundidad la total amplitud del proceso, debemos indicar que éste abarcó cambios en el proceso productivo, en las formas hegemónicas de socialización, en la modalidad predominante en la que se gestionaban y compartían los riesgos sociales, en las relaciones laborales, en la representación y mediación política, en la organización de la vida familiar, en la agregación de demandas colectivas y en las formas de participación y lucha política, entre otros. Robert Castel destaca -por sobre la función redistributiva, que generalmente se le atribuye- el carácter "protector" que desempeñó el Estado social al brindar, a los miembros de la sociedad salarial, un acceso a la propiedad social, es decir, al poner a su disposición las protecciones que anteriormente sólo procuraba la propiedad privada. ${ }^{1}$ De este modo, este autor sostiene que a partir de la década de 1970, cuando comienza a producirse el resquebrajamiento del sistema de protecciones, se advierte como efecto un sostenido aumento de las incertidumbres y la inseguridad.

En el presente artículo buscamos indagar en las articulaciones que se presentan en la coyuntura sociopolítica actual de la Argentina entre las formas de problematización de

\footnotetext{
${ }^{1}$ CASTEL, Robert. La inseguridad social. ¿Qué es estar protegidos? Buenos Aires: Manantial, 2004.
} 
Polit. crim. Vol. 6, No 11 (Junio 2011), Art. 1, pp. 1 - 18.

[http://www.politicacriminal.cl/Vol_06/n_11/Vol6N11A1.pdf]

la cuestión social, las políticas preventivas de control del delito y las reorientaciones en materia de políticas sociales, especialmente, en el campo de las políticas sociales asistenciales.

En un trabajo que ya se ha convertido en un clásico de la sociología reciente de la metamorfosis de las relaciones asalariadas, Robert Castel señalaba que la cuestión social consiste en la inquietud fundamental acerca de la capacidad que tiene una sociedad de conjurar el riesgo de su fractura o fracturas. ${ }^{2}$ Sin embargo, tal como ha afirmado Estela Grassi:

"la cuestión social no se presenta como tal (como contradicción y virtualmente fractura), sino que, en cada época, se particulariza en problemas sociales que son, a la vez, la expresión hegemónica del modo como se interroga, interpreta, resuelve, ordena y canaliza la misma" (énfasis en el original). ${ }^{3}$

Por lo tanto, se impone el estudio del modo en que dicha cuestión se actualiza en cada contexto. En el caso de la Argentina contemporánea, entendemos que el tratamiento hegemónico del problema de la (in)seguridad articula tramas argumentativas que condensan y son solidarias con la producción de modos específicos de experiencia e inteligibilidad de la fractura social constitutiva de las sociedades capitalistas. La actual configuración histórica de la cuestión social escenifica privilegiadamente a la pobreza como condición social a ser gobernada donde se dirime la tensión identitaria víctimavictimario, asimismo se presenta el espacio público como dimensión espacial en la cual se debe garantizar la presencia y circulación de los portadores de la condición de ciudadanos y limitar la de aquellos excluidos de tal condición.

De esta manera, entendemos que en la última década se ha consolidado en la Argentina una tendencia que construye progresivamente a la problemática de la (in)seguridad como núcleo de la tensión constante de la cuestión social. Consecuentemente, la (in)seguridad se instaura como constitutiva del orden social. ${ }^{4}$ En este sentido, esta particular problematización de la cuestión social, que presenta al problema de la (in)seguridad como expresión hegemónica del modo en que es interpretada, diagnosticada y encauzada la potencial fractura social, brinda las condiciones de emergencia de nuevas intervenciones que construyen específicas relaciones entre las nociones de "delito" y "condiciones de vida". En este artículo abordaremos estas intervenciones desde dos ángulos: en primer lugar, en el campo de las políticas sociales, las reorientaciones paulatinas hacia intervenciones dirigidas sobre diversos grupos caracterizados por sus diferentes niveles de seguridad y peligrosidad; y, en segundo lugar, las políticas de prevención social del delito que conjugan explícitamente intervenciones propias del campo de la política social con intervenciones propias del campo de la política criminal.

\footnotetext{
2 CASTEL, Robert. Las metamorfosis de la cuestión social. Una crónica del salariado. Buenos Aires: Paidós, 2004.

${ }^{3}$ GRASSI, Estela. Políticas y problemas sociales en la sociedad neoliberal. La otra década infame (I). Buenos Aires: Espacio Editorial, 2003, p. 22.

${ }^{4}$ PEGORARO, Juan Segundo. "Las relaciones sociedad-Estado y el paradigma de la inseguridad". Delito y Sociedad. Revista de Ciencias Sociales, número 9-10, Buenos Aires: Oficina de Publicaciones del CBC. (1997), pp. 51-63.
} 
AYOS, Emilio; DALLORSO, Nicolás. "(In)seguridad y condiciones de vida en la problematización de la cuestión social: Políticas sociales y políticas de prevención social del delito".

\section{Mutación del "par seguridad-inseguridad" en el campo de las políticas sociales.}

El régimen de acumulación heredado del fin de la Segunda Guerra Mundial posibilitó que se estructurara la "forma Estado" y su relación con el orden social en el marco del paradigma de la seguridad. ${ }^{5}$ En una etapa verdaderamente excepcional del desarrollo del capitalismo, el aumento de la producción global posibilitó la inclusión de importantes sectores de la población bajo el paraguas de la seguridad social. De este modo, a partir de la segunda posguerra en la Argentina, la extensión de las seguridades sociales, que se expresaron en la masificación y consolidación del modelo bismarckiano de esquema contributivo, convivieron con una sostenida debilidad de las instituciones democráticas, la proscripción de la fuerza política mayoritaria y la intolerancia a la disidencia política. Así, a partir de mediados de la década de 1940, al ya existente desarrollo del sistema universalista de educación pública lo siguió la creación de un nuevo sistema universalista de salud pública, basado tanto en la estructuración de un sistema hospitalario a nivel nacional y en la creación de centros médicos ambulatorios como en el crecimiento y consolidación del sistema de obras sociales. Asimismo, se impulsaron las seguridades en materia previsional a través de la expansión de la cobertura legal a todos los sectores laborales y la adopción de un sistema de reparto, que asumía la idea de que el sistema previsional debía ser un instrumento para la redistribución del ingreso y se sustentaba en una lógica implícita de un pacto intergeneracional por el cual los trabajadores activos sostenían a la pasividad por vejez, invalidez o fallecimiento de un familiar. Este modelo de protección social mutua, asociado al trabajo como propiedad social, construyó un entramado que proveyó seguridad en las diferentes etapas de la vida y frente a circunstancias en las que el individuo no puede solventar su reproducción y la de su familia a través de su trabajo.

A partir de mediados de la década de 1970 durante la última dictadura militar, pero, especialmente, a lo largo de la década de 1990, con el gobierno menemista, se instaura un régimen de acumulación de características neoliberales por el cual se socavaron los basamentos de aquel paradigma de la seguridad que protegía las condiciones de vida de los trabajadores.

En lo que respecta al mercado de trabajo, se impuso la flexibilidad fundamentada en la teoría económica neoclásica; es decir, se propugnó eliminar "rigideces" institucionales que impedirían el libre funcionamiento de la demanda para remunerar al trabajo según su aporte a la productividad marginal. Los mecanismos de flexibilidad numérica, que se refieren a los instrumentos que le permiten al sector empresarial ampliar su capacidad para contratar y "descontratar" de acuerdo a las necesidades del proceso de producción, se extendieron durante toda la década. Con las reformas en la legislación laboral operadas en el período, muchos de estos mecanismos fueron legalizados precarizando la relación laboral. Para ejemplificar algunos de los tipos de contrataciones que utilizaron estos mecanismos de reducción de la protección laboral podemos mencionar: el período de prueba, las diversas modalidades de empleo temporario, los contratos de aprendizaje, las pasantías y las distintas clases de subcontratación. El desempleo abierto y la masificación de esta heterogenización de la contratación laboral han resquebrajado la concertación entre actores económicos claves puesto que los sindicatos vieron

\footnotetext{
${ }^{5}$ PEGORARO, "Las relaciones", cit. nota n 4.
} 
Polit. crim. Vol. 6, No 11 (Junio 2011), Art. 1, pp. 1 - 18.

[http://www.politicacriminal.cl/Vol_06/n_11/Vol6N11A1.pdf]

fuertemente restringido su accionar y los empresarios se vieron posibilitados a obtener mayores ventajas eludiendo los compromisos corporativos.

En materia de protección previsional, la reforma del sistema de jubilaciones y pensiones llevada adelante en 1993 implicó la privatización de la seguridad pública existente para la tercera edad. El esquema previsional reformado adoptó un sistema de múltiples pilares, con un fuerte énfasis en un pilar obligatorio de capitalización y ahorro individual bajo gestión privada. Este componente del sistema reformado provee beneficios a cada uno de los pasivos con la cantidad de primas que ellos mismos hayan pagado en el pasado, de tal forma que, no existen ganancias netas por el hecho de participar del sistema. Esta lógica estricta del seguro, al preservar los índices diferenciales según la condición de ahorro individual, imposibilita cualquier tipo de intervención redistributiva. De esta forma, la modalidad de capitalización individual acaba con el principio de solidaridad intergeneracional que operaba en los regímenes de reparto. Asimismo, la profunda crisis que el mercado laboral argentino arrastra desde la década de 1990 (flexibilización laboral, contrataciones encubiertas, despidos masivos, desempleo abierto y prolongado, etc.) hace especialmente difícil el cumplimiento de las condiciones de asignación de los beneficios jubilatorios -30 años mínimos de aportes- a grandes sectores de la población económica activa, en especial, a las mujeres, los desempleados y los trabajadores del sector informal. ${ }^{6}$

Es en este sentido que en las últimas dos décadas, en materia de políticas de gobierno de la cuestión social, hemos asistido a un tránsito desde políticas que regulaban el andamiaje de protecciones jurídicas asociadas a la relación capital/trabajo, a partir de intervenciones sobre las relaciones salariales -entendiéndolas como vectores centrales de la integración social-, hacia un régimen neoliberal de políticas sociales, reduciéndose el rol de estas últimas al de ser subsidiarias en materia de pobreza. A partir de la utilización del dispositivo de la focalización como principio articulador, las políticas de "combate contra la pobreza" buscan amortiguar el impacto de los programas de ajuste en los grupos más vulnerables de la población. En este marco, se promueve el otorgamiento de recursos compensatorios a quienes, y sólo a quienes, ya sea a título definitivo o provisorio, no alcanzan un umbral suficiente de subsistencia. Es en este sentido que, el carácter asistencial de las políticas sociales, en el período reseñado, ha adquirido una marcada centralidad, relegando a un segundo plano el interés por las causas de los procesos de producción de exclusión social. ${ }^{7}$

En esta misma dirección que, desde las indagaciones referidas al control social, Pegoraro plantea la presencia de un paradigma de la inseguridad para aludir al hecho de

\footnotetext{
${ }^{6}$ En noviembre de 2008, el Congreso Nacional sancionó un proyecto ley impulsado por el Poder Ejecutivo Nacional que implica una virtual contra-reforma del sistema previsional al reinstaurar el sistema de reparto y eliminar las administradoras privadas de fondos de jubilaciones y pensiones. Dado que el cambio es aún muy reciente, no se toman en cuenta los efectos de dicha transformación en este escrito. Para un mayor desarrollo de esta reforma ver Beccaria, Alejandra y Danani, Claudia. "El proceso de reforma previsional argentina 2003-2008: instituciones y sentidos". Ponencia presentada en las $X$ Jornadas Argentinas de Estudios de Población, 2009. Disponible en http://www.produccion.fsoc.uba.ar/aepa/xjornadas/pdf/50.pdf

7 EZCURRA, Ana María. ¿Qué es el neoliberalismo? Evolución y límites de un modelo excluyente. Buenos Aires: Lugar Editorial, 1998. ANDRENACCI, Luciano, "Algunas reflexiones en torno a la cuestión social y la asistencialización de la intervención social del Estado en la Argentina contemporánea”. En ANDRENACCI, Luciano (organizador) Cuestión social y política social en el Gran Buenos Aires. Buenos Aires: Ediciones Al Margen / UNGS, 2002.
} 
AYOS, Emilio; DALLORSO, Nicolás. "(In)seguridad y condiciones de vida en la problematización de la cuestión social: Políticas sociales y políticas de prevención social del delito".

que la mencionada transformación invierte el sistema de seguridades que había caracterizado al régimen de acumulación heredado del fin de la Segunda Guerra Mundial, puesto que extiende la mercantilización de relaciones sociales, anteriormente basadas en el servicio y la solidaridad,

"creando una inseguridad creciente como la menor atención pública a la salud, una escasa política de vivienda [...] una disminución en el sostenimiento de la educación pública y una anulación o reducción drástica de los derechos laborales; y además el empobrecimiento de más de dos tercios de la sociedad". ${ }^{8}$

Este proceso -detenidamente analizado en los estudios sobre la cuestión social y la exclusión social- ${ }^{9}$ ha tenido como efecto la degradación de la condición social de determinados grupos y la consecuente concentración de la vulnerabilidad y percepción de inseguridad en ellos.

En la forma en que se ha instalado, en el último tiempo, la problemática de la (in)seguridad en Argentina, los potenciales ofensores aparecen fuertemente vinculados con los grupos vulnerabilizados a partir de la desestructuración de los sistemas de protección social, especialmente, los jóvenes, pobres, sin familia, sin empleo y con escasos o nulos lazos con los centros educativos. En este sentido, tanto la adopción de "estrategias de acercamiento", por ejemplo la focalización, así como la creciente acentuación del carácter asistencialista por parte de las intervenciones estatales poswelfaristas han sido condiciones de posibilidad para la reactualización de la cuestión de las "clases peligrosas" en el diseño e implementación de las políticas sociales, puesto que crecientemente las políticas sociales asistenciales recortan sus objetos de intervención sobre los mismos grupos señalados como potenciales ofensores por los discursos hegemónicos del control social.

Aun cuando algunos autores como Norberto Alayón y Estela Grassi ${ }^{10}$ señalan que no toda intervención de asistencia social a situaciones de emergencia y/o a grupos sociales específicos debe ser conceptuada en términos de asistencialismo, la asistencia social tradicionalmente ha sido un sector de políticas estigmatizantes porque sus destinatarios se identificaron, alternativamente, como "vagos", "débiles", "oportunistas", "marginales", "inadaptados" o "carecientes". En la Argentina, a partir de la instauración del régimen social de acumulación en la década de 1990, las intervenciones sociales del Estado (especialmente, los programas compensatorios focalizados en la pobreza) se dirigieron crecientemente a identificar y asistir -solamente- a los más débiles, distinguiéndolos de aquellos que pudiesen procurarse su bienestar y protección a través del mercado, sustituyéndose, así, un marco general de derechos y garantías exigibles por acciones volátiles, coyunturales y aleatorias. ${ }^{11}$

Si bien el concepto de focalización no ha estado siempre vinculado a los discursos neoliberales de ajuste estructural y reforma del Estado, sino que, en la década de 1970,

\footnotetext{
${ }^{8}$ PEGORARO, "Las relaciones", cit. nota $n^{\circ} 4$, p.54.

9 FITOUSSI, Jean Paul y ROSANVALLON, Pierre. La era de las desigualdades. Buenos Aires: Manantial, 1996. CASTEL, "Las metamorfosis", cit. nota $\mathrm{n}^{\circ} 2$.

${ }^{10}$ ALAYÓN, Norberto. Asistencia y asistencialismo. ¿Pobres controlados o erradicación de la pobreza? Buenos Aires: Humanitas, 1992. GRASSI, "Politicas y problemas sociales", cit. nota n³.

${ }^{11}$ Cfr. GRASSI, "Políticas y problemas sociales", cit. nota $\mathrm{n}^{\mathrm{o}} 3$.
} 
Polit. crim. Vol. 6, No 11 (Junio 2011), Art. 1, pp. 1 - 18.

[http://www.politicacriminal.cl/Vol_06/n_11/Vol6N11A1.pdf]

dicha noción formó parte de los debates acerca de las políticas redistributivas, excediendo el marco de las políticas sociales, sin duda, a partir de los diagnósticos de la crisis fiscal en los años 1980, la focalización se incorpora como herramienta preeminente de selectividad del gasto social y de la lucha contra la pobreza extrema, en el marco de las políticas de ajuste fiscal y "racionalización" del gasto social (cfr. Sojo 1990). Articulándose con las críticas -no sólo liberales- de las prácticas homogenizantes y normalizantes de las intervenciones welfaristas, el discurso neoliberal ha incorporado el requisito de la focalización como criterio de eficiencia, equidad y racionalidad en la gestión de la política social.

El carácter estigmatizante de estas políticas aparece sumamente visible tanto en el proceso de la definición de la población-objetivo como en la instrumentación en terreno de la selectividad. En el primer caso, como afirma Tenti Fanfani ${ }^{12}$ los individuos pasan de ser estadísticamente pobres a ser socialmente vistos y tratados como pobres, con la consecuente institucionalización y legalización de las desigualdades sociales. En el segundo, se produce una particular relación social entre los agentes estatales de la asistencia social (ya sean formuladores de las políticas o profesionales de la asistencia) y su "clientela" donde se disputan el "derecho a merecer asistencia": los primeros desconfiando y estando atentos ante la presencia de "abusadores" y los otros dando pruebas de tal merecimiento.

El escenario abierto por la emergencia del paradigma de la inseguridad también se caracteriza por esta creciente extensión de intervenciones de carácter puntual, local y situativo, tanto en el ámbito de las políticas sociales así como en el campo de las políticas de prevención del delito.

Como señalamos más arriba, los grupos que han visto desestructurados sus sistemas de protecciones sociales y, especialmente, los jóvenes de sectores populares, son aquellos que concentran en sí todas las percepciones de amenaza y son los grupos que, en el mejor de los casos, se constituyen en poblaciones-objetivo de intervenciones asistenciales y reciben prestaciones sociales en tanto son caracterizados como grupos "en riesgo". Asimismo, ante la urgencia que imponen los consensos punitivos en materia de control de la criminalidad, se consolida, en las esferas gubernamentales y en la opinión pública, una mayor disposición a justificar las intervenciones orientadas al bienestar de sectores vulnerados sólo en términos de la prevención del delito, con el consecuente riesgo de criminalizar la política social. De este modo, se niega un marco general de derechos y garantías exigibles, que pudiese habilitar un reclamo e, incluso, la organización colectiva en derredor de derechos sociales no cumplidos y se argumenta el otorgamiento de recursos compensatorios con el fin de la protección de la seguridad del resto de los ciudadanos.

De todas maneras, siguiendo a Castel, ${ }^{13}$ entendemos que no hay que oponer inseguridad $\mathrm{y}$ protecciones como si éstas pertenecieran a registros opuestos de la experiencia colectiva: la sensación de inseguridad percibida socialmente no es sinónimo de ausencia de protecciones. Existe "un desfase entre una expectativa socialmente construida de

12 TENTI FANFANI, Emilio. "Representación, delegación y acción colectiva en comunidades urbanas pobres". En: LUMI, Susana; GOLBERT, Laura; TENTI FANFANI, Emilio. La mano izquierda del estado. La asistencia social según los beneficiarios. Madrid-Buenos Aires: Miño y Dávila-Ciepp, 1993.

${ }^{13}$ CASTEL, "La inseguridad social" cit. nota $\mathrm{n}^{\circ} 1$. 
AYOS, Emilio; DALLORSO, Nicolás. "(In)seguridad y condiciones de vida en la problematización de la cuestión social: Políticas sociales y políticas de prevención social del delito".

protecciones y las capacidades efectivas de una sociedad dada para ponerlas en funcionamiento". " ${ }^{14}$ Se plantea, entonces, una paradoja entre seguridad y protección. Aun cuando los programas protectores puedan cumplir parcialmente sus objetivos se producen, por una parte, decepción - por la imposibilidad de una total seguridad- y, por otra parte, mayor incertidumbre porque al dominar ciertos riesgos, emergen otros nuevos.

\section{Cuestión social, (in)seguridad y prevención del delito.}

A partir de fines de la década de 1970 comienza a producirse lo que se ha considerado como un cambio paradigmático en cuanto a las estrategias orientadas al control de la criminalidad. ${ }^{15}$ En el contexto general de crisis de los Estados de Bienestar, y en particular del entramado de instituciones ligadas al control del delito que en ellos se había gestado, conocido como el "welfarismo penal" o el "complejo penal-welfare", ${ }^{16}$ emerge el desarrollo de la prevención extra penal del delito, es decir, la prevención proactiva, para diferenciarla de la intervención puramente reactiva propia del sistema penal. ${ }^{17}$

Las formas que adquieren estas intervenciones no penales son variadas y hacen de la política criminal (conjunto formado por estas políticas y las penales propiamente dichas) un concepto complejo, indeterminado, que contiene diversas técnicas concretas, dependiendo de la posición teórica que lo sustente. ${ }^{18}$ Estos desarrollos en acciones proactivas, que en conjunto forman lo que se conoce como "nueva prevención" comparten dos características: por un lado, el ingreso de la comunidad local como protagonista de este tipo de estrategias, reflejado en la pluralidad de organismos que participan en sus distintos niveles de implantación. ${ }^{19}$ Por otro lado, la extensión de las acciones preventivas más allá de las que se dirigen a conductas que infringen la ley penal, alcanzando a aquellas denominadas incivilités, ${ }^{20}$ no formalmente delictivas. ${ }^{21}$ Otra de las particularidades que distinguen a este tipo de intervenciones se vincula con el intento de verificación empírica de los resultados de la intervención, es decir, la intención de evaluar mediante instrumentos técnicamente idóneos el impacto o el grado de alcance del objetivo planteado. ${ }^{22}$

\footnotetext{
${ }^{14}$ CASTEL, "La inseguridad social" cit. nota $\mathrm{n}^{\mathrm{o}} 1, \mathrm{p} .13$.

15 SOZZO, Máximo. "Seguridad Urbana y Técnicas de Prevención del Delito", en: Cuadernos de Jurisprudencia y Doctrina Penal, número 10, (2000). Buenos Aires.

${ }^{16}$ GARLAND, David. La cultura del control: crimen y orden social en la sociedad contemporánea. Barcelona: Editorial Gedisa, 2005.

${ }^{17}$ Es importante esta salvedad, puesto que a lo largo de su historia, siempre se le adjudicó a la prisión funciones preventivas, aunque también desde su nacimiento surgieron múltiples voces que denunciaron su constante fracaso. Cfr. FOUCALT, Michel. Vigilar y Castigar. México: Siglo XXI, 1999. BARATTA, Alessandro. "Política Criminal: entre la política de seguridad y la política social", en: CARRANZA, E. (coord.): Delito y seguridad de los habitantes. México DF: Siglo XXI, 1997.

${ }^{18}$ BARATTA, "Política Criminal", cit. nota $\mathrm{n}^{\circ} 17$.

19 PAVARINI, Massimo. Castigar al enemigo. Criminalidad, exclusión e inseguridad. Quito: Flacso Ecuador, 2009.

${ }^{20}$ Las incivilidades (o incivilités en su término francés) hacen referencia a conductas y situaciones no formalmente delictivas que afectan la convivencia en un territorio.

${ }^{21}$ BARATTA, "Política Criminal", cit. nota n ${ }^{\circ}$ 17. O’MALLEY, Pat, "Riesgo, poder y prevención del delito". En: Revista Delito y Sociedad, número 20, (2004). Buenos. Aires: UNL.

${ }^{22}$ PAVARINI, "Castigar al enemigo", cit. nota no 19.
} 
Polit. crim. Vol. 6, No 11 (Junio 2011), Art. 1, pp. 1 - 18.

[http://www.politicacriminal.cl/Vol_06/n_11/Vol6N11A1.pdf]

Más allá de estos elementos distintivos, la característica que configura como "nueva" a la nueva prevención es fundamentalmente la proliferación de estrategias que se ubican por fuera del ámbito de lo penal, es decir, que son extra-penales y que, frente a la concepción pasiva e indirecta de la prevención penal, oponen un modelo que intenta configurarse como directo y pro-activo. $^{23}$ No obstante lo cual, si bien las criminologías correccionalistas que proliferaron con la formación del complejo "penal-welfare",24 a finales del siglo XIX y principios del XX confiaban en la capacidad de "la reforma social" para atenuar la comisión de delitos, es decir, en los mecanismos extra-penales, el control del delito gubernamental continuó centrándose en el sistema penal. De esta manera, la efectiva proliferación de estrategias extra penales dentro de la política criminal se muestra como uno de los elementos distintivos de la nueva prevención.

Al interior del conjunto de intervenciones que son denotadas con la noción de "prevención del delito", dada su amplitud e intensa heterogeneidad, surgen numerosas formas de clasificación y de condensación de las mismas en tipos o estrategias diferentes. Una de estas clasificaciones propone diferenciar entre los tipos de prevención primaria, secundaria y terciaria. ${ }^{25} \mathrm{El}$ primero se caracterizaría por orientarse sobre las "condiciones criminógenas" en un contexto físico o social dado, sin referencia a prácticas ilegales anteriores. El segundo estaría conformado por medidas dirigidas a "grupos en riesgo" delictivo. Por último, la prevención terciaria se distinguiría por intervenir luego de la comisión de delitos, con el fin de prevenir futuros incidentes. Este esquema también se ha complejizado introduciendo las dimensiones de las víctimas y el contexto. Así, podríamos diferenciar intervenciones preventivas, primarias, secundarias y terciarias, sobre los autores (potenciales o no), las víctimas (también, potenciales o no) y los contextos. Otras tipologías instan a diferenciar las estrategias preventivas en relación con una dimensión temporal de su ingerencia (antes, durante o luego del acto), el objeto de su intervención (los comportamientos de los sujetos o la modificación del contexto social) y su carácter defensivo (sobre los síntomas o emergentes) u ofensivo (sobre las causas). ${ }^{26}$

Sin embargo, existe, como dijimos, una forma preponderante de estructurar las diferencias entre las distintas intervenciones concretas que se desarrollan bajo la noción de "prevención del delito" y es la que separa o diferencia dos grandes grupos: la prevención situacional-ambiental y la prevención social. En el desarrollo histórico de las intervenciones de prevención se han identificados también otras dos formas que serán desarrolladas más adelante: la prevención comunitaria y la prevención del desarrollo (developmental crime prevention). ${ }^{27}$

Podemos decir que la táctica situacional-ambiental se caracteriza fundamentalmente por estar orientada hacia las víctimas potenciales y esta orientación se evidencia a partir de organizar todo un conjunto diverso de intervenciones sobre las situaciones y los ambientes para reducir las oportunidades delictivas. El marco conceptual de referencia del cual parten estas intervenciones hace hincapié, no en el delito entendido como una

\footnotetext{
${ }^{23}$ SELMINI, Rossella. "La prevención: estrategias, modelos y definiciones en el contexto europeo". En: Urvio. Revista Latinoamericana de Seguridad Ciudadana. número 6, (2009). Quito: Flacso Ecuador.

${ }^{24}$ GARLAND, "La cultura del control", cit. nota $\mathrm{n}^{\circ} 16$.

${ }^{25}$ SOZZO, "Seguridad Urbana", cit. nota no 15. SELMINI, "La prevención”, cit. nota no 23.

${ }^{26}$ SELMINI, "La prevención", cit. nota n 23.

27 SOZZO, "Seguridad Urbana", cit. nota n' 15. O’MALLEY "Riesgo", cit. nota n" 21. SELMINI, "La prevención”, cit. nota $n^{\circ} 23$.
} 
AYOS, Emilio; DALLORSO, Nicolás. "(In)seguridad y condiciones de vida en la problematización de la cuestión social: Políticas sociales y políticas de prevención social del delito".

predisposición individual, sino como consecuencia de factores que favorecen las oportunidades delictivas de actores presentados como racionalmente motivados. ${ }^{28}$ Estas corrientes, que David Garland conceptualizó como criminologías de la vida cotidiana, integran las teorías del delito como elección racional, la teoría de las actividades rutinarias y el delito como producto de la oportunidad. Su supuesto básico es que el delito no responde a una disposición individual particular, a una patología o anormalidad, sino que es un evento que se encuentra inscripto en las rutinas de la vida social contemporánea. Es decir, el delito es un fenómeno normal, producto de ciertas rutinas, ciertas oportunidades que un individuo racional evalúa para su accionar. De alguna manera, estas criminologías expresan el pasaje desde una noción de rehabilitación, propia de las criminologías del "penal-welfare", hacia la prevención, mediante la reducción de la disponibilidad de oportunidades, la modificación de rutinas y el aumento del control situacional. Esto supone el descentramiento del individuo ofensor y sus condiciones de existencia por la noción de "evento delictivo" y "situaciones criminógenas". ${ }^{29}$

Por su parte, el modelo de prevención social se caracteriza por intentar influir en las supuestas causas sociales-estructurales que explicarían los comportamientos delictivos; es decir, a diferencia de los anteriores modelos de prevención situacional-ambiental, se orienta fundamentalmente hacia los potenciales ofensores. Este modelo contiene resabios de las interpretaciones positivistas sobre la "reforma social" como método ex - ante de prevención, aunque la influencia de esta corriente se diluyó en la discusión sobre las causas del delito avanzada la segunda posguerra. Dentro del mundo anglosajón (EE.UU., Canadá - angloparlante - y en menor medida en Gran Bretaña) son fragmentarias las implementaciones de la táctica social ante el predominio del modelo situacionalambiental. En general, las tácticas de prevención social en el mundo anglosajón, se dirigieron mayormente hacia los jóvenes, como potenciales ofensores en tanto "grupo de riesgo". " A diferencia del anglosajón, en el contexto francés (Francia y luego la Canadá francoparlante) la táctica social se convirtió en un elemento fundamental de la política criminal en los años finales de la década del setenta. El diagnóstico sobre las causas del delito dentro de este ámbito se centró en el problema de la exclusión social: las intervenciones, entonces, tendieron a proponer estrategias de integración social para los grupos que habían quedado en los márgenes de las redes de interacción social: en este caso, los jóvenes, los desocupados y los inmigrantes. ${ }^{31}$

Uno de los puntos centrales de debate en torno a este tipo de intervenciones es su especificidad, fundamentalmente, en relación con el campo de las políticas sociales, lo que constituye el punto nodal de nuestra indagación. Dado que más adelante nos referiremos a esta relación, nos interesa poner de manifiesto aquí que este debate se estructura entre quienes proponen una visión "amplia" de las políticas de prevención social, asimilándolas al conjunto de intervenciones sociales del Estado, y entre quienes buscan delimitar su especificidad a partir de identificar lo particular de sus objetos de intervención y de sus fines propuestos. Algunos representantes de esta última posición, distinguen dos orientaciones internas dentro de esta estrategia: una ligada a las

28 BARATTA, "Política Criminal", cit. nota $n^{\circ}$ 17. SOZZO, "Seguridad Urbana", cit. nota no 15. SELMINI, "La prevención", cit. nota n 23.

${ }^{29}$ GARLAND, "La cultura del control", cit. nota n 16.

${ }^{30}$ SOZZO, "Seguridad Urbana", cit. nota no 15.

${ }^{31}$ BARATTA, "Política Criminal", cit. nota n 17. SOZZO, "Seguridad Urbana", cit. nota no 15. 
Polit. crim. Vol. 6, No 11 (Junio 2011), Art. 1, pp. 1 - 18.

[http://www.politicacriminal.cl/Vol_06/n_11/Vol6N11A1.pdf]

motivaciones individuales y otra, al contexto social. De esta manera, tienden a diluir la prevención social entre estrategias hacia el autor potencial, la "prevención del desarrollo" y las estrategias orientadas a las condiciones sociales comunitarias, la "prevención comunitaria del delito". ${ }^{32}$

Como vimos, la noción de lo local es una de las dimensiones distintivas de la proliferación contemporánea de la prevención del delito. Y en este marco aparece la importancia de la interpelación a la comunidad en estas políticas. La autonomización de la prevención comunitaria como un tipo diferente de táctica preventiva estaría dada por la forma en que estas políticas construyen a la comunidad como sujeto activo de la intervención y no sólo como su "lugar" u objeto de realización. Dentro de estas estrategias de responsabilización de los ciudadanos, ${ }^{33}$ conviven tanto intervenciones que promueven una idea de "comunidad defensiva" frente a los potenciales ofensores, como intentos de desarrollar participación comunitaria y nuevas formas de solidaridad social. Esto marca el grado en que la prevención comunitaria puede pensarse como una forma compuesta, que engloba tanto medidas de prevención social como estrategias situacionales. ${ }^{34}$ Las intervenciones que usualmente se realizan bajo la denominación de prevención comunitaria pueden condensarse en estos tres enfoques: la organización de la comunidad, como movilización de los residentes para el fortalecimiento del control social informal; la defensa de la comunidad, bajo variadas formas de autoprotección, vigilancia comunitaria o vinculación con la reforma urbana y arquitectónica y por último, el desarrollo de la comunidad, ligada al mejoramiento de las condiciones sociales de vida. ${ }^{35}$

Como hemos mencionado, también se ha autonomizado un cuarto tipo de intervención preventiva, diferenciada por el énfasis en las motivaciones individuales a la hora de construir sus estrategias: la prevención del desarrollo. Ésta hace referencia al desarrollo, en el curso de la vida individual, de aquellas motivaciones, entendidas como condiciones criminógenas. Y en esta vertiente es particularmente importante la noción de "factores de riesgo": podrían diferenciarse los factores individuales (bajo autocontrol, agresividad, escasa capacidad de planificación, etc.), los factores familiares (fallas en el ejercicio de la paternidad, conflicto, abusos, abandonos, etc.) y los factores socio-económicos (condiciones de privación, degradación comunitaria, influencia de los "pares", etc.). ${ }^{36} \mathrm{Y}$, aunque como vemos, habría puntos de indistinción con la estrategia social, la diferenciación radica en el énfasis sobre los procesos de conformación de estas estructuras motivacionales a nivel individual, durante el desarrollo de un curso de vida.

Las formas en las que estas diferentes estrategias se combinaron en intervenciones concretas en el contexto europeo permiten establecer dos modelos de políticas de prevención del delito. Por un lado, el modelo anglosajón, caracterizado por los siguientes elementos: una prevalencia fuerte de la prevención situacional por sobre la social; el protagonismo de las agencias policiales; el énfasis en las potenciales víctimas, tanto en su necesidad de protección como en el sentido de su responsabilización, a partir de mecanismos de involucramiento de la comunidad local en funciones de vigilancia; una impronta managerial y, por último, aunque se observan procesos de responsabilización

\footnotetext{
${ }^{32}$ SELMINI, "La prevención", cit. nota n ${ }^{\circ} 23$.

${ }^{33}$ GARLAND, "La cultura del control", cit. nota $\mathrm{n}^{\circ} 16$.

${ }^{34}$ SELMINI, "La prevención", cit. nota n 23.

35 SELMINI, "La prevención", cit. nota n 23.

${ }^{36}$ SELMINI, "La prevención", cit. nota n 23.
} 
AYOS, Emilio; DALLORSO, Nicolás. "(In)seguridad y condiciones de vida en la problematización de la cuestión social: Políticas sociales y políticas de prevención social del delito".

de otros actores institucionales o no institucionales, un control centralizado de los programas preventivos por parte del Estado. Por otro lado, el modelo francés o continental se distingue por: el predominio de la táctica social por sobre la situacional; el intento de inscribir las intervenciones en el ámbito local, al nivel de los alcaldes; una limitada responsabilización de las víctimas como de la comunidad; un modelo menos dirigista, enfocado en la búsqueda de colaboraciones entre el plano nacional y local (por ejemplo en los "contratos de prevención" en Francia durante la década de 1980); participación de la policía a partir del modelo de "proximidad" con la ciudadanía. ${ }^{37}$

Sin embargo, en los últimos años se observa una tendencia general hacia la centralización de la estrategia situacional-ambiental que pareciera hacer más borrosa la distinción que aquí presentamos entre el modelo anglosajón y el franco-continental. Y también, surge con fuerza la estrategia "del desarrollo" ante una declinación de las estrategias típicamente sociales. Esta declinación se observa tanto en la pérdida de relevancia de las medidas preventivas al interior de los programas preventivos, como en la "baja intensidad" de sus intervenciones: sólo algunas medidas asistenciales de corto alcance. ${ }^{38}$

También en la política criminal argentina se observan transformaciones en el contexto del neoliberalismo en la década de 1990. En general, se podría describir en aquella un desplazamiento hacia las estrategias de prevención ex - ante del delito. Sin embargo, consideramos que la idea de "desplazamiento" no capta la complejidad de estas mutaciones, porque, si bien la orientación preventiva extra-penal ha surgido con fuerza, ello no ha comportado una retracción del modelo penal, sino todo lo contrario: se configuraría una polarízación de la política criminal y no un desplazamiento. ${ }^{39}$ El acontecimiento que expresa la emergencia de la orientación preventiva en la Argentina es, sin duda, la puesta en marcha del Plan Nacional de Prevención del Delito en el año 2000. Por supuesto, las formas en las que surgen estas intervenciones responden a procesos histórico-políticos específicos, propios de los escenarios nacionales. El proceso en el que surge la prevención del delito en la Argentina es significativo en este sentido: el Plan Nacional de Prevención del Delito (PNPD en adelante) puesto en marcha por el gobierno nacional puede entenderse como producto de conflictos y debates acerca de la cuestión de la (in)seguridad en el contexto político argentino en aquellos años. Es fundamental remarcar que en el período que va desde 1998 hasta el año 2000 dicha cuestión alcanza niveles de politización inéditos ${ }^{40}$ y la incorporación de estrategias preventivas aparece como un posicionamiento "progresista" frente a las posturas punitivistas ligadas a la noción "mano dura" frente al delito. La figura más fuerte ligada a este último posicionamiento fue, sin duda, el por entonces gobernador de la provincia de Buenos Aires por el Partido Justicialista, Carlos Ruckauf.

\footnotetext{
${ }^{37}$ SOZZO, "Seguridad Urbana", cit. nota no 15. SELMINI, "La prevención", cit. nota no 23.

${ }^{38}$ SELMINI, "La prevención", cit. nota n 23.

${ }^{39}$ RANGUGNI, Victoria. "Transformaciones del Estado y Políticas de Control del Delito en la Argentina de los '90". En: MUÑAGORRI y PEGORARO (comps.) La relación seguridad-inseguridad en centros urbanos de Europa y América Latina, Estrategias, Politicas, Actores, Perspectivas, Resultados. Madrid: Editoria Dickinson, 2004.

${ }^{40}$ SOZZO, Máximo. "Globalización y control del delito. Algunas reflexiones exploratorias" en: Revista de la Facultad de Ciencias Jurídicas y Sociales, número 3, año 3, (2003). Santa Fe: Ediciones UNL.
} 
Polit. crim. Vol. 6, No 11 (Junio 2011), Art. 1, pp. 1 - 18.

[http://www.politicacriminal.cl/Vol_06/n_11/Vol6N11A1.pdf]

Específicamente, en torno a los procesos de vinculación entre las formas de problematización de la cuestión social y las intervenciones que desde el campo de la política criminal intentan "resolver" la (in)seguridad, el surgimiento del PNPD abría nuevas dinámicas: éste preveía una línea de intervención social, que en el año 2001 cristalizaría en la creación del Programa Comunidades Vulnerables. Y, si bien el desarrollo del PNPD fue limitado y su lugar dentro de la política criminal argentina fue marginal, este programa logró durante algunos años -fundamentalmente a partir del año 2003- establecerse en varias unidades político-territoriales. Además, es destacable que en los años finales del PNPD (es dado de baja durante el primer trimestre de 2008) el Programa Comunidades Vulnerables fue la única intervención que el PNPD administraba.

Este programa se implementó en diferentes zonas de la Ciudad de Buenos Aires y del Gran Buenos Aires, además de hacerlo en otras provincias: Río Negro, Neuquén, Chubut, Mendoza y Santa Fe. En su formulación, el programa define su acción como "focalizada en barrios de alta vulnerabilidad social" y abocado a dos ejes de acción interrelacionados: a) la prevención de la violencia social y b) la prevención del delito. La metodología de trabajo es definida en torno al modelo de Investigación Acción Participativa (IAP), el que supone la participación de las poblaciones-objeto en las distintas instancias de la intervención. El programa se organiza a partir de tres áreas: la jurídica, que dispone de orientación y derivación jurídica en materia penal y familiar; el área vincular que prevé acciones (para contrarrestar deficiencias en el funcionamiento de las relaciones vinculares originadas en las distintas situaciones de vulnerabilidad) como ser, entre otras, actividades culturales, artísticas, recreativas y deportivas, talleres de prevención de la violencia y el uso indebido de drogas y, por último, el área de microemprendimientos, que estimula su organización, entendiéndolos como una forma de reducir las "situaciones de riesgo".

Nos interesa destacar que en su puesta en práctica, el Programa Comunidades Vulnerables estableció una novedosa vinculación entre los campos de la política criminal y la política social en la Argentina. En su desarrollo, este programa acopló como parte de su intervención los planes asistenciales administrados por el Ministerio de Trabajo y Seguridad Social, desde el año 2003, con el Programa de Empleo Comunitario (PEC). El otorgamiento de estos planes fue una parte fundamental de las estrategias de prevención social del delito implementadas por el Programa Comunidades Vulnerables, ya que fue el mecanismo de transferencia de ingresos utilizado como "contraprestación" por participar en el programa.

Esta imbricación entre intervenciones sociales y control del delito que pone en juego el Programa Comunidades Vulnerables es un lugar privilegiado para analizar los modos de articulación entre la prevención social del delito y las formas particulares en que la cuestión social es problematizada, fundamentalmente en su orientación hacia la temática de la (in)seguridad. En este sentido nos interesa la particular vinculación que las estrategias de prevención social establecen con el campo de las políticas sociales. Es el "carácter problemático" 41 de esta relación el que genera interrogantes fundamentales de nuestra investigación. Cuando calificamos de "problemática" esta ligazón entre prevención social del delito y políticas sociales nos referimos a la dificultad de trazar una línea clara de diferenciación entre los dos campos, en cuanto a las prácticas a través

\footnotetext{
${ }^{41}$ BARATTA, "Política Criminal”, cit. nota no 17 . SOZZO, "Seguridad Urbana", cit. nota no 15.
} 
AYOS, Emilio; DALLORSO, Nicolás. "(In)seguridad y condiciones de vida en la problematización de la cuestión social: Políticas sociales y políticas de prevención social del delito".

de las cuales los programas en cuestión se realizan. ${ }^{42}$ Las implicancias políticas de esta superposición de campos son trascendentes, puesto que al ponerse en práctica estos planes, es probable que la política criminal reencuentre a estos sujetos como objetos de una política social sin que por ello sean valorados en la finalidad de dicha política: el objetivo consiste en aumentar la seguridad de otros ciudadanos. En este sentido, creemos que existe la posibilidad de que se generen procesos que implican una criminalización de las políticas sociales $^{43}$ entendiendo por ésta, la creciente incorporación de objetivos de política criminal en intervenciones que históricamente correspondieron al campo de las políticas sociales. Esta transformación se plasma en la adopción de este tipo de políticas por parte de las agencias de política criminal, imprimiéndole su propia dinámica.

Así, dentro de esta nueva racionalidad, los grupos en los cuales se enfocan los programas se separan de aquellos verdaderos "beneficiarios" de las intervenciones: la poblaciónobjetivo de los programas son los sectores sociales de potencial peligrosidad, mientras que aquellos que se ven beneficiados con las intervenciones son otros grupos socialmente más protegidos. Esta última consideración nos remite nuevamente al concepto de "criminalización", centrado ahora en los procesos de construcción social que intervienen en la definición de lo delictivo. Fueron los aportes del interaccionismo simbólico y la etnometodología, especialmente las teorías del etiquetamiento, los que nos brindaron, desde el paradigma de la reacción social, las primeras herramientas teóricas para analizar los mecanismos de interacción social que se ponen en juego en el "etiquetamiento" y estigmatización de ciertas conductas y perfiles (etarios, étnicos, de género, etc.) como criminales. Luego, la criminología crítica incorporará el análisis político e histórico de las relaciones de poder definitorias dentro de estos procesos en un nivel estructural ${ }^{44}$ y ligará el concepto de "criminalización" al de "selectividad": la política penal como encargada de administrar diferencialmente los ilegalismos. ${ }^{45}$ Esta administración supone una criminalización de las clases subalternas, conjugado con un solapamiento de los delitos del poder, ${ }^{46}$ puesto que del conjunto total de los ilegalismos, sólo algunos son construidos como delitos por las agencias estatales y esta construcción está directamente ligada a las estructuras políticas y sociales establecidas en una sociedad determinada.

Es en esta línea que podemos analizar los programas de prevención del delito a partir de la categoría de "criminalización": estos corren el riesgo de reproducir la selectividad de la política penal, al no considerar en sus intervenciones los delitos conocidos como de "cuello blanco", económicos, ecológicos, de corrupción; los vinculados a las fuerzas de seguridad y otras agencias estatales; así como tampoco las situaciones de violencia y opresión que sufren mujeres y niños en el ámbito doméstico. La política penal tiende a abocarse únicamente al restringido conjunto de conductas que aparecen estereotipadas en el sentido común como "criminalidad tradicional" y que tienen como atributo el asignar

\footnotetext{
${ }^{42}$ BARATTA, "Política Criminal", cit. nota $\mathrm{n}^{\mathrm{o}} 17$.

${ }^{43}$ BARATTA, "Política Criminal", cit. nota n 17. SOZZO, "Seguridad Urbana", cit. nota no 15.

44 PAVARINI, Massimo. Control y Dominación. México: Siglo XXI Editores, 1983. BARATTA,

Alessandro. Criminología Crítica y Crítica del Derecho Penal. Buenos Aires: Siglo XXI Editores, 1986.

${ }^{45}$ FOUCALT, "Vigilar y Castigar", cit. nota n 17.

46 PEGORARO, Juan Segundo. "Inseguridad Urbana y los Delitos de la Autoridad y el Poder". En: SOZZO, Máximo. (Ed.) Seguridad Urbana: Nuevos Problemas, Nuevos Enfoques. Santa Fe: UNL, 1999.
} 
Polit. crim. Vol. 6, No 11 (Junio 2011), Art. 1, pp. 1 - 18.

[http://www.politicacriminal.cl/Vol_06/n_11/Vol6N11A1.pdf]

regularmente los papeles de víctima y victimario: este último reservado para los sectores más vulnerables, expuestos a la marginalidad y la pobreza.

Partimos de afirmar que en los programas de prevención del delito se reproducen las estructuras de desigualdad al considerar como potenciales ofensores de la ley penal sólo a ciertos grupos sobre la base de sus condiciones sociales y económicas de vida y al estructurar políticas públicas bajo esta orientación. Es en este sentido que creemos que existe el riesgo de que los programas de prevención social del delito generen procesos de criminalización de la pobreza, ${ }^{47}$ para lo cual resulta pertinente la observación del momento lógico de mayor implicación de los agentes institucionales: el de la formulación y gestión directa de las políticas. Cabe señalar que este momento no agota el proceso de "criminalización", ni ningún otro proceso de constitución de sujetos sociales, pero permite reconstruir el sentido y las relaciones fundantes de esas intervenciones.

\section{Reflexiones finales.}

En este artículo hemos presentado algunos de los elementos fundamentales de las transformaciones históricas que en el nivel de las intervenciones políticas expresan cierta tendencia contemporánea hacia la progresiva problematización de la cuestión social como cuestión de la (in)seguridad frente al delito, haciendo referencia específicamente al caso argentino contemporáneo. En este sentido, entendemos que el tratamiento hegemónico del problema de la (in)seguridad articula tramas argumentativas que condensan y son solidarias con la producción de modos específicos de experiencia e inteligibilidad de esta fractura social constitutiva de las sociedades capitalistas. Por esto nos hemos referido en este artículo a las transformaciones en intervenciones sociales: las que se ponen en marcha dentro del propio campo de las políticas sociales así como también las que son conjugadas con políticas preventivas del delito en un espacio ambiguo con la política criminal. Consideramos que muchas de estas transformaciones pueden ser entendidas si las comprendemos como parte de un proceso por el cual la forma hegemónica de construcción de la (in)seguridad tiñe los modos en las que es recreada la cuestión social.

En el campo de las políticas sociales hemos observado una mutación relevante en la fundamentación de las intervenciones sociales que han abandonado un paradigma vinculado a la protección integral de las condiciones de vida de la masa de trabajadores y han adoptado una disposición tendencial a privilegiar la fundamentación de intervenciones de carácter puntual y coyuntural sobre grupos clasificados de acuerdo a sus niveles de riesgo y peligrosidad. En este sentido, a medida que las políticas sociales han adoptado progresivamente una orientación dirigida a la "lucha contra la pobreza" se transforman los fundamentos, los objetivos, la metodología y los objetos que habían caracterizado a las intervenciones sociales en el contexto del Estado social. En paralelo a este proceso, el problema de la (in)seguridad se ha instalado en la escena política y social constituyéndose en un tema prioritario del funcionamiento del sistema político argentino. Como hemos señalado más arriba, el problema de la (in)seguridad, tal como se plantea

${ }^{47}$ BARATTA, "Política Criminal", cit. nota $\mathrm{n}^{\mathrm{o}}$ 17. WACQUANT, Loïc, Las cárceles de la miseria. Buenos Aires: Manantial, 2000. PEGORARO, Juan Segundo, "Teoría Sociológica y Delito Organizado: El Eslabón Perdido" en: Encrucijadas UBA. Revista de la Universidad de Buenos Aires, número 19. (2002). Buenos Aires: UBA. 
AYOS, Emilio; DALLORSO, Nicolás. "(In)seguridad y condiciones de vida en la problematización de la cuestión social: Políticas sociales y políticas de prevención social del delito".

en su tratamiento hegemónico, escenifica privilegiadamente a la pobreza como condición social a ser gobernada donde se dirime la tensión identitaria víctima-victimario. De este modo, cobra especial relevancia el papel que adquiere la política social, especialmente la de carácter asistencial orientada a la "lucha contra la pobreza", en la actual configuración histórica de la cuestión social.

A su vez, observamos estos procesos en la emergencia de las intervenciones que se inscriben dentro de la llamada nueva prevención del delito, específicamente en las estrategias de prevención social. El desarrollo de estas tácticas de prevención se vincula también al proceso de transformación histórica que expresó la crisis del Estado de Bienestar durante la década de 1970, en particular la progresiva desarticulación del "complejo penal-welfare", y lo fundamentalmente nuevo de esta nueva prevención fue su construcción a partir de intervenciones extra-penales. La intervención sobre lo social fue uno de los caminos recorridos fuera de lo penal. De esta manera, la reorientación de la política criminal hacia lo social aparece como uno de los lugares fundamentales para observar estos procesos de redefinición de la cuestión social, haciendo hincapié en el carácter fundamentalmente problemático de la relación entre estrategias preventivas y políticas sociales. Como hemos mencionado al comenzar este artículo en la Argentina contemporánea se reactualiza la constitutiva fractura de la sociedad a partir de tramas argumentativas que privilegian e instalan prioritariamente el problema de la (in)seguridad en la agenda pública. En este sentido, hemos buscado realizar una aproximación a la especificidad que asume el entrecruzamiento de intervenciones en el campo de la política social y la política criminal. Este cruce en un cuadro de indiferenciación más allá de etiquetas institucionales cobra especial relevancia puesto que brinda las condiciones de posibilidad para la extensión de procesos de criminalización de las políticas sociales y, en lo que se refiere a la construcción de sujetos sociales, la generalización de las bases materiales que posibilitan la legitimación de los procesos de criminalización de la pobreza. 
Polit. crim. Vol. 6, № 11 (Junio 2011), Art. 1, pp. 1 - 18.

[http://www.politicacriminal.cl/Vol_06/n_11/Vol6N11A1.pdf]

\section{BIBLIOGRAFÍA}

ALAYÓN, Norberto, Asistencia y asistencialismo. ¿Pobres controlados o erradicación de la pobreza? Buenos Aires: Humanitas, 1992.

ANDRENACCI, Luciano, "Algunas reflexiones en torno a la cuestión social y la asistencialización de la intervención social del Estado en la Argentina contemporánea", en: ANDRENACCI, Luciano, (organizador) Cuestión social y politica social en el Gran Buenos Aires. Buenos Aires: Ediciones Al Margen / UNGS, 2002.

BARATTA, Alessandro, Criminología Crítica y Crítica del Derecho Penal. Buenos Aires: Siglo XXI Editores, 1986.

, "Política Criminal: entre la política de seguridad y la política social", en: CARRANZA, E. (coord.): Delito y seguridad de los habitantes. México DF: Siglo XXI, 1997.

BECCARIA, Alejandra / DANANI, Claudia, "El proceso de reforma previsional argentina 2003-2008: instituciones y sentidos". Ponencia presentada en las $X$ Jornadas Argentinas de Estudios de Población, 2009. Disponible en http://www.produccion.fsoc.uba.ar/aepa/xjornadas/pdf/50.pdf

CASTEL, Robert, Las metamorfosis de la cuestión social. Una crónica del salariado. Buenos Aires: Paidós, 2004.

, La inseguridad social. ¿Qué es estar protegidos? Buenos Aires: Manantial, 2004.

EZCURRA, Ana María, ¿Qué es el neoliberalismo? Evolución y límites de un modelo excluyente. Buenos Aires: Lugar Editorial, 1998.

FITOUSSI, Jean Paul / ROSANVALLON, Pierre, La era de las desigualdades. Buenos Aires: Manantial, 1996.

FOUCALT, Michel, Vigilar y Castigar. México: Siglo XXI, 1999.

GARLAND, David, La cultura del control: crimen y orden social en la sociedad contemporánea. Barcelona: Editorial Gedisa, 2005.

GRASSI, Estela, Politicas y problemas sociales en la sociedad neoliberal. La otra década infame (I). Buenos Aires: Espacio Editorial, 2003.

O'MALLEY, Pat, "Riesgo, poder y prevención del delito", Revista Delito y Sociedad, número 20, (2004). Buenos. Aires: UNL.

PAVARINI, Massimo, Control y Dominación. México: Siglo XXI Editores, 1983.

, Castigar al enemigo. Criminalidad, exclusión e inseguridad. Quito: Flacso Ecuador, 2009.

PEGORARO, Juan Segundo, "Las relaciones sociedad-Estado y el paradigma de la inseguridad". Delito y Sociedad. Revista de Ciencias Sociales, número 9-10 (1997), Buenos Aires: Oficina de Publicaciones del CBC, pp. 51-63.

, "Inseguridad Urbana y los Delitos de la Autoridad y el Poder", en: SOZZO, Máximo. (Ed.) Seguridad Urbana: Nuevos Problemas, Nuevos Enfoques. Santa Fe: UNL, 1999.

"Teoría Sociológica y Delito Organizado: El Eslabón Perdido", Encrucijadas UBA. Revista de la Universidad de Buenos Aires, número 19 (2002). Buenos Aires: UBA.

RANGUGNI, Victoria, "Transformaciones del Estado y Políticas de Control del Delito en la Argentina de los '90", en: MUÑAGORRI/PEGORARO (comps.) La relación seguridad-inseguridad en centros urbanos de Europa y América Latina, 
AYOS, Emilio; DALLORSO, Nicolás. “(In)seguridad y condiciones de vida en la problematización de la cuestión social: Políticas sociales y políticas de prevención social del delito".

Estrategias, Politicas, Actores, Perspectivas, Resultados. Madrid: Editoria Dickinson, 2004.

SELMINI, Rossella, "La prevención: estrategias, modelos y definiciones en el contexto europeo", Urvio. Revista Latinoamericana de Seguridad Ciudadana. número 6, (2009). Quito: Flacso Ecuador.

SOZZO, Máximo, "Seguridad Urbana y Técnicas de Prevención del Delito", Cuadernos de Jurisprudencia y Doctrina Penal, número 10 (2000), Buenos Aires.

,"Globalización y control del delito. Algunas reflexiones exploratorias", Revista de la Facultad de Ciencias Jurídicas y Sociales, número 3, año 3 (2003). Santa Fe: Ediciones UNL.

TENTI FANFANI, Emilio, "Representación, delegación y acción colectiva en comunidades urbanas pobres", en: LUMI, Susana / GOLBERT, Laura / TENTI FANFANI, Emilio, La mano izquierda del estado. La asistencia social según los beneficiarios. Madrid-Buenos Aires: Miño y Dávila-Ciepp, 1993.

WACQUANT, Loïc, Las cárceles de la miseria. Buenos Aires: Manantial, 2000. 